\title{
Author Correction: Scalable synthesis of silicon-nanolayer-embedded graphite for high-energy lithium-ion batteries
}

Minseong Ko, Sujong Chae, Jiyoung Ma, Namhyung Kim, Hyun-Wook Lee, Yi Cui and Jaephil Cho

Correction to: Nature Energy https://doi.org/10.1038/nenergy.2016.113, published online 8 August 2016.

In this Article, the authors should have declared the following competing interest: "The authors have a patent (Korean Patent application no. 1020140128496, registered 2 June 2016) related to the processes described in this Article".

Published online: 9 March 2020

https://doi.org/10.1038/s41560-020-0587-8

(C) The Author(s), under exclusive licence to Springer Nature Limited 2020 